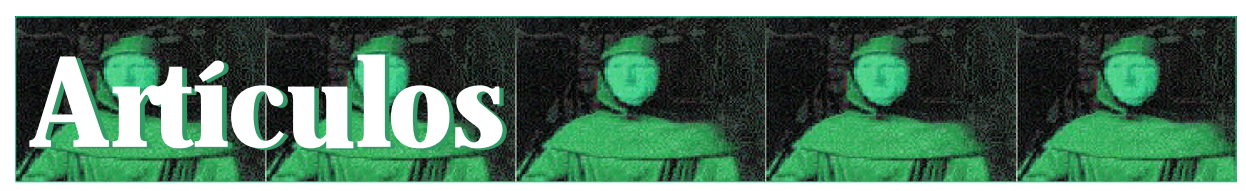

\title{
Modelación matemática de la actividad bibliotecaria: una revisión
}

\author{
SALVADOR GORBEA PORTAL \\ CentroUniversitariodeInvestigacionesBibliotecológicas \\ Circuito Interior,CiudadUniversitaria,C.P.04510,México,D .F. \\ Tel: (525) 623- 0360, Fax: (525)550- 7461 \\ E-mail:portal@ servidor.unam.mx
}

Trabajo recibido

el 15 de agosto de 1997

Trabajo acepta-

do el 8 de enero

de 1998

\section{RESUMEN}

Sepresentaunarevisión del desarro llo al can zado porlamo delaciónmatemá ticaen laactividad biblio tecaria, apartirdelasprin cipalesaportacio nesreali zadas en este campo y entendidas como consecuencia de las relaciones interdisciplinarias que se manifiestan en trelas mate máticasylas cien cias bi blio teco ló gicaydelainformación,ycomo partedelamatematizacióndelconocimiento científico, en particular el social. Se hace especial énfasis en las con tribuciones deRan ganathan yen suvisiónal distinguirtalesaportaciones como un cuerpo de conocimiento al cual denominó Librametry.

\begin{abstract}
ABST RACT
A review is presented on the development attained by mathematical modeling in library science with emphasis on its main contributions to the field. These are seen as consequences of the interdisciplinary relationships existing between mathematics, and library and information science, and as part of the "mathematization" of scientific knowledge, particularly its social aspects. Also underlined are Ranganathan's contributions and his vision in identifying the body of knowledge which he named Librametry.
\end{abstract}

\section{MATEMATIZACIÓN DEL CONOCIMIENTO CIENTÍFICO}

Uno de los factores que ha caracterizado el desarrollo de la ciencia contemporánea, sin lu garadu das, eslapenetración deméto dosymo delosmatemáticos, en forma reproductora, en todas las esferas del conocimiento,incluyendo por supuesto el de las ciencias sociales. 
Esta práctica, en ocasiones, ha estado dirigida a dar cientificidad al discurso intelectual de esta rama del conocimiento y, en otras, a formalizar leyes que identifiquen el comportamiento de fenómenos y procesos sociales, que sin la ayuda de las matemáticas no hubiera sido posible identificar.

Este proceso que cada vez toma mayor fuerza en las ciencias en general y las sociales en particular se conoce como la matematización di conocimiento centífico el cual, a nuestro criterio, en las ciencias sociales se manifiesta por medio de dos tendencias fundamentales: la primera, referida a la representación o simulación matemática de fenómenos y procesos sociales reales, lo que presupone el diseño y creación de modelos matemáticos específicos que los representen y, la segunda, a la utilización de modelos cuantitativos ya establecidos y generalizados en las matemáticas en la medición, análisis, interpretación y regulación de tales fenómenos y procesos.

La primera tendencia ha propiciado el surgimiento de leyes y teorías, señaladas por Puchet, pertenecientes a las matemáticas aplicadas, pero que se han desarrollado en función de las ciencias sociales, tales como:

* Teoría de la optimación

* Teoría de la elección social

* Teoría de los juegos y de las decisiones

* Teorías de las series temporales

* Teorías estadísticas del análisis de datos y del muestreo

* Teoría de la inferencia econométrica.

En este sentido, el autor destaca que, aunque algunas de estas teorías tienen un origen matemático clásico, han encontrado su verdadero desarrollo y comprobación en ciencias sociales como por ejemplo la economía (Puchet, 1993, pp.114- 117).

La segunda tendencia ha posibilitado la aplicación de métodos matemáticos especiales tales como la investigación de operaciones, mediante ecuaciones algebraicas 0 métodos de programación lineal, o no lineal, estocásticos, entre otros, así como métodos y análisis estadísticos, muy importantes en las ciencias sociales,

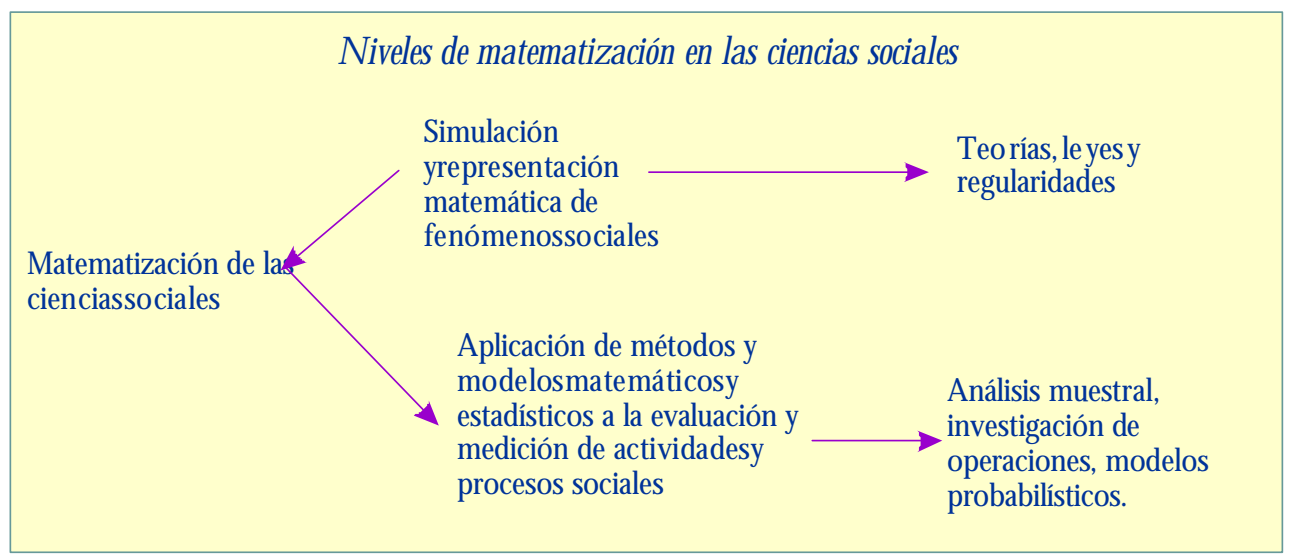


especialmente en la sociología, entre los que se pueden mencionar los de regresión, correlación, factorial, discriminantes, etcétera.

La matematización del conocimiento científico implica la reproducción desu lengrajeen dro, detipo simbólice, quelolleaa a un mayor nived deabstración Este planteamiento, aportado por Redondo Botella (1990, p. 51) en su libro, entraña que este proceso no está presente cuando las matemáticas se aplican como simples herramientas cuantitativas, sino cuando a partir de métodos y modelos matemáticos se puede enriquecer el lenguaje formal de las ciencias sociales, es decir, cuando existe una verdadera traducción de leyes, procesos o fenómenos sociales al lenguaje matemático, que coadyuve a identificar comportamientos y regularidades antes no identificadas sin la ayuda de las matemáticas, de ahí que diversos autores sostienen que sólo se alcanza el estatuto de ciencia cuando se han matematizado los enunciados de su discurso.

Pero este proceso de matematización requiere determinados postulados y relaciones filosóficas y matemáticas, así como la necesaria relación entre la dialéctica objetiva y la subjetiva y entre lo cuantitativo y lo cualitativo, por lo que el mismo requiere que se manifieste a la luz de los vínculos interdisciplinarios que se establecen entre las matemáticas y las ciencias sociales y que constituye un indiscutible método para la aproximación y enriquecimiento mutuo.

También debe entenderse en este proceso que el conocimiento matemático, a diferencia del estadístico que conlleva la medición y confrontación de argumentos empíricos, debe estar dirigido hacia la formulación de argumentos teóricos y en esta medida, según Puchet:

[...]deben ser forma representativa por excelencia de los enunciados de todo discurso científico, sin que esto conduzca a la interpretación de que la cientificidad de una ciencia particular, dependa de manera decisiva y determinante del grado de matematización que en ella se alcance (Puchet, 1993, p.118).

La producción de información/ conocimiento es un fenómeno social que no escapa al proceso de aplicación de las matemáticas, se evidencia con absoluta claridad, a través de las relaciones interdisciplinarias entre ésta y las disciplinas científicas dedicadas al estudio de tal fenómeno.

Todo lo anterior no implica que el proceso de matematización de las ciencias y la aplicación de métodos y modelos matemáticos a las actividades y práctica sociales, como el que acabamos de describir, sea privativo de matemáticos; todo lo contrario, constituye un nivel de profundización y especialización de los estudiosos vinculados a los temas de análisis de estas disciplinas, actividades y fenómenos, entre los que se pueden mencionar, en la esfera ciencia/ información, a los especialistas en información, bibliotecólogos, bibliógrafos, cienciólogos y filósofos e historiadores de la ciencia, entre otros.

$\mathrm{Si}$ observamos el comportamiento de la información/ conocimiento, como objeto de estudio, a través de un fenómeno social como lo es la generación y uso 
de la información, así como de las actividades que están presentes en el ciclo social de la información, podemos identificar que la matematización de las ciencias sociales (que son las que estudian este fenómeno) ha enriquecido el lenguaje formal de estas disciplinas, lo cual ha dado lugar a una nueva línea de investigación conocida como la metría de la información/ conocimiento, así como el surgimiento de nuevas especialidades métricas, vinculadas a las disciplinas bibliológico/ informativa.

Proceso de matematización del sistema de conocimientos bibliológico/ informativos en relación con los fenómenos que estudia y las especialidades métricas que se han derivado de éste

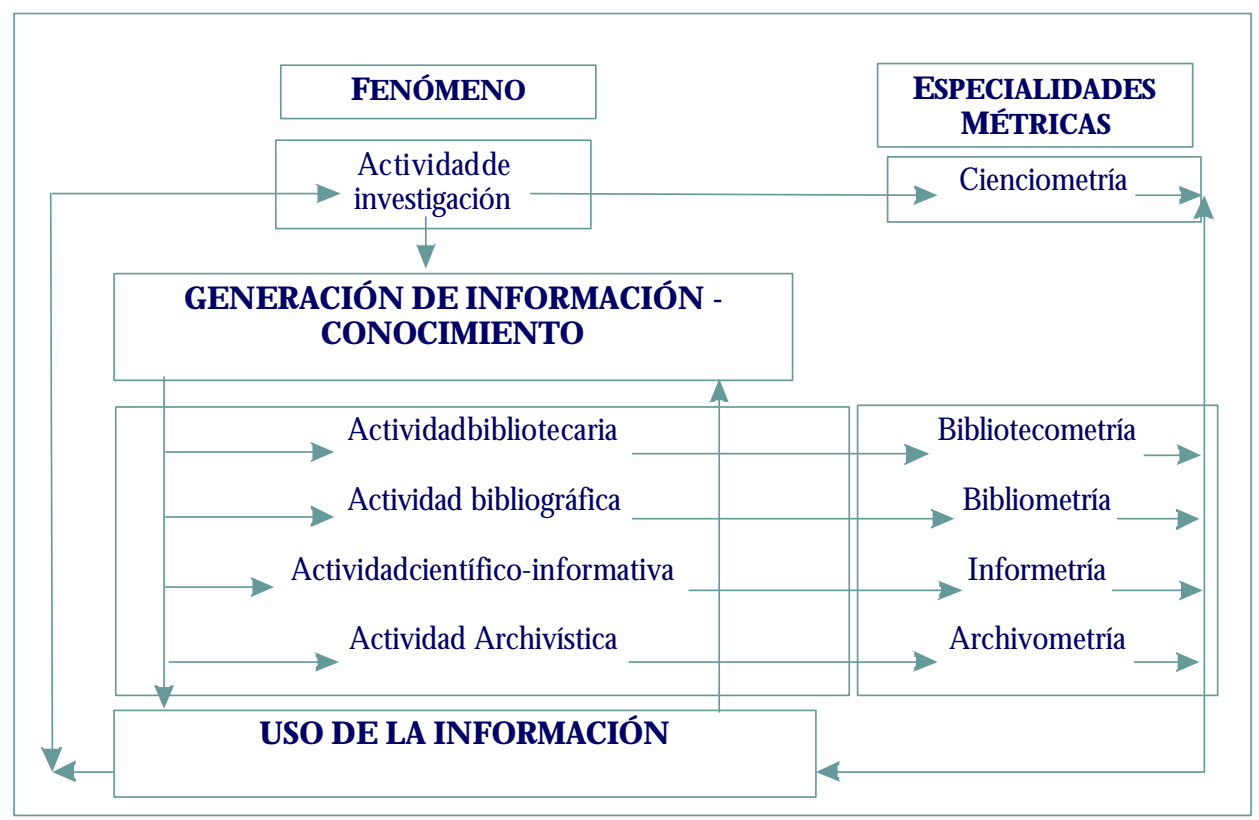

La observación anterior echa por tierra criterios de algunos especialistas, que limitan todo el proceso de matematización que se viene operando en los sistemas de conocimientos, a la simple utilización de los métodos y modelos matemáticos como una herramienta más de las actividades científica, bibliológico/ informativa y otras de la esfera social.

Ese enfoque reducido, el cual niega el carácter reproductivo que el proceso de matematización alcanza en las relaciones interdisciplinarias que se dan entre las matemáticas y las ciencias sociales, no llega a percibir la posibilidad de que el comportamiento de las regularidades de la información científico técnica (crecimiento, concentración/ dispersión, obsolescencia, uso), como fenómeno social, pueda ser 
reproducido mediante un modelo matemático que caracterice e interprete también el sistema de leyes que lo rige.

El proceso de matematización de las disciplinas que estudian los fenómenos sociales no sólo se manifiesta a través del enriquecimiento del lenguaje formal de estas disciplinas y del surgimiento de nuevas especialidades métricas vinculadas a tales fenómenos, sino que se aprecia también en la aplicación de métodos y modelos matemáticos a soluciones prácticas, llamadas a resolver el desarrollo de las actividades de cada una de ellas. Con ello se demuestra, por una parte, la capacidad que tienen las matemáticas de inducir y estructurar nuevas especialidades métricas con sustento empírico y, por la otra, la de las disciplinas científicas, entre éstas las bibliológico/ informativas, por asimilar tales conocimientos y enriquecer los enunciados de su discurso.

En tal sentido se observa la utilización de métodos y modelos matemáticos para el análisis de indicadores científicos que caracterizan el comportamiento de los sistemas científicos, por medio de sus indicadores de entrada y salida y, el obligado análisis de costo/ beneficio que apunta a la eficiencia de los sistemas científicos, además de la evaluación de investigadores e instituciones científicas.

Conocidos en nuestro medio son también, por un lado, aquellos modelos que han resultado de la observación del comportamiento de regularidades sobre el crecimiento, concentración/ dispersión, entre otras, de los flujos de información compilados en los diversos repertorios bibliográficos o colecciones de bibliotecas, $y$, por otro, el utilizado para evaluar el comportamiento de bibliotecas en desarrollo y otros que facilitan el análisis, mantenimiento y uso de colecciones de bibliotecas con el afán de ayudar a la toma de decisiones en la gestión de las actividades bibliotecaria y de información. En ambos sentidos se obtienen resultados diferentes como los que se observan a continuación:

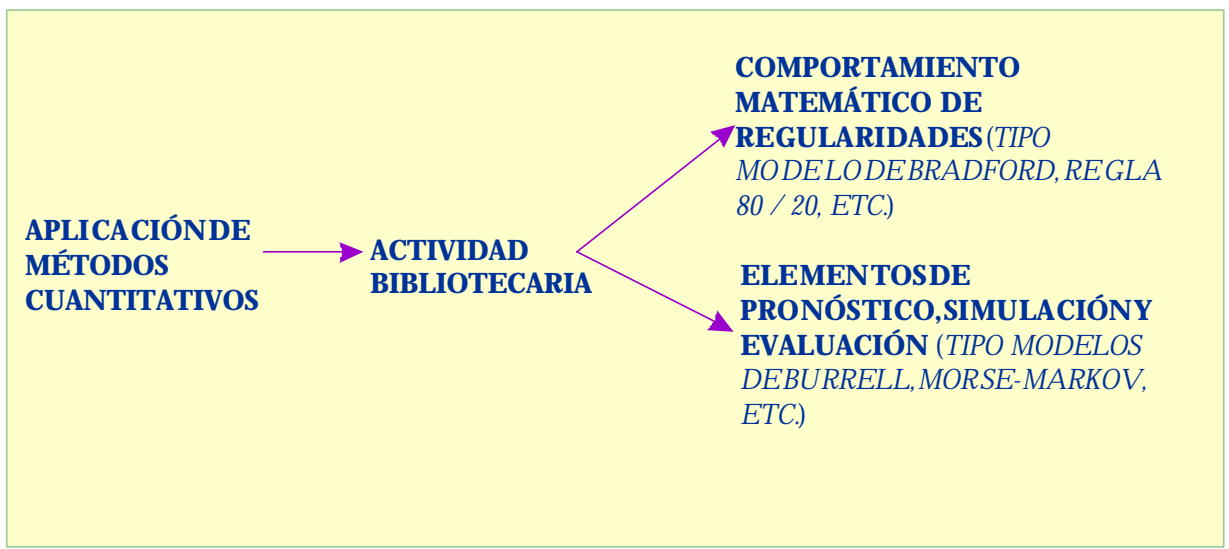

Es precisamente a esta última práctica que se dedicó un Seminario-Taller, es decir, a la aplicación de métodos y modelos matemáticos y estadísticos a la 
actividad bibliotecaria, centrada por supuesto, en el entorno de la matematización de la información/ conocimiento, como fenómeno social, y dentro de éste, a la acumulación, sistematización, preservación y difusión de la información, columna vertebral de nuestra actividad profesional. El presente trabajo y los subsiguientes fueron presentados y debatidos en este Seminario/ Taller.

En la actualidad, el bibliotecólogo, al igual que cualquier científico, requiere de información y datos métricos mensurables para poderse enfrentar a la toma de decisiones en la gestión de muy diversos procesos y operaciones unitarias que se desarrollan cotidianamente en la actividad bibliotecaria, pero para ello deberá contar con la formación matemática que se lo permita, sin embargo, es oportuno señalar que no siempre la matemática está presente en la dimensión necesaria en los mapas curriculares cursados por los egresados en bibliotecología. De aquí la idea de agregar al contenido de este número monográfico de Investigacón Biblicte coóġaun artículo en el que se presentan algunas reflexiones sobre la importancia de las matemáticas en los contenidos curriculares de la carrera de Bibliotecología y Ciencia de la Información.

En este sentido, Gómez y Peralta (1995, p. 40) destacan la unidad entre las matemáticas y la ciencia, así como su vinculación con los nuevos programas de estudio. Estas autoras señalan, con muy atinada razón, la importancia de la enseñanza de las matemáticas en la formación científica, y ejemplifican cómo un experimento científico de tipo dicotómico puede ser diseñado con la posibilidad de obtener datos métricos mensurables que permitan el conocimiento de relaciones y regularidades de fenómenos gracias a las matemáticas.

El bibliotecólogo al utilizar el método científico realiza observaciones a su objeto de estudio, que le aportan información también de carácter dicotómico, 0 sea de todo o nada, sí o no, que lo ponen en la misma disyuntiva que la de un científico. Si se establece una analogía con el ejemplo de las autoras antes citadas, podemos analizar en nuestra actividad las interrogantes siguientes: ¿se compra 0 no determinado documento?; ¿se aumenta el número de asientos en el área de consulta?; ¿se amplía el horario de servicios? entre otras preguntas que también pueden ser formuladas en la búsqueda de información para la toma de decisiones referente a estos aspectos. Todos ellos determinados en última instancia por el factor financiero, que en ocasiones se convierte en otra pregunta dicotómica en una segunda etapa de la decisión ¿se cuenta o no con los recursos financieros para tomar la decisión?

Mediante la información obtenida a partir de una interrogante de tipo dicotómico se puede determinar si es necesaria o no cada una de estas observaciones, pero no podrá conocer en qué medida o dimensión se debe manifestar tal decisión.

De ahí que si el bibliotecólogo del sistema bibliotecario necesita información sobre el comportamiento de las necesidades identificadas, deberá analizar el conjunto de variables que intervienen en cada decisión que ha de tomarse, de manera 
que pueda obtener datos métricos que contribuyan a conocer la estructura y dimensión de cada fenómeno analizado.

Por esto se tendrá que recurrir a las matemáticas para obtener un conocimiento más completo del fenómeno que quiere analizar, es decir, para poder decidir no sólo si aumenta el número de asientos, sino también en cuántos los aumenta; si amplía el horario de servicio, pero en cuánto y en qué sesiones.

Esta práctica, sobre el conocimiento de los fenómenos, procesos y tareas de la actividad bibliotecaria, requiere cada vez, con mayor urgencia, de la utilización de conocimiento originado en las matemáticas, para poder formular cada uno de ellos en términos cuantitativos, así como conocer el comportamiento de sus variables y las relaciones que se establecen entre éstas.

Resulta comprensible entonces, entender la definición que los matemáticos dan a su disciplina, cuando afirman que la matemática: "es la ciencia de los modelos, considerada como un ente que de cuyo comportamiento se puede derivar el comportamiento de un fenómeno o sistema" (Herrera, 1997).

D e esta definición de las matemáticas, como la ciencia de los modelos, emerge la interrogante ¿qué es un modelo matemático? Según Luisa Redondo, "un modelo matemático es el prototipo de forma idealizada, por lo tanto es un objeto abstracto, que por analogía y síntesis representa el fenómeno o proceso y para conseguirlo toma las características que se consideren más importantes de una realidad objetiva, entre las que deben estar presentes, directa 0 indirectamente, las esenciales del caso concreto" (Redondo Botella, 1990, p. 97).

Un acercamiento y entendimiento de los bibliotecólogos y especialistas de la información a estos conceptos abre ante sí un campo infinito en la evaluación, análisis, sistematización y difusión de la información en las bibliotecas y otras instituciones de información. De incursionar el bibliotecólogo en la práctica y sistematización de las matemáticas a la gestión bibliotecaria, contribuiría con ello a enriquecer el lenguaje formal de su disciplina y alcanzaría mayores niveles de eficiencia en la institución en que se desempeña como profesional.

\section{LA MODELACIÓN MATEMÁTICA DE LA ACTIVIDAD BIBLIOTECARIA}

Si revisamos toda la literatura disponible y especializada en la utilización de mé todos cuantitativos a los procesos y tareas que se realizan en una institución bibliotecaria o de información, podemos inferir, con extrema claridad, que la modelación matemática ofrece al bibliotecólogo o especialista en información una herramienta adicional para identificar comportamientos en una biblioteca.

Sin embargo, sería limitado entender que estos comportamientos pudieran referirse a comportamientos puramente matemáticos, debido a que en ocasiones, por medio de esta valiosa herramienta se pueden llegar a identificar elementos de pronóstico y simulación de todos y cada uno de los procesos y tareas que se 
realizan en la actividad bibliotecaria y con ello al surgimiento de una ley o postulado teórico, que sin el uso de la matemática no se hubiera resuelto.

Uno de los primeros modelos que se identificó en la actividad bibliotecaria fue el comportamiento matemático sobre la distribución de artículos por títulos de revistas, es decir, el modelo matemático de Bradford, en 1934. Con este modelo, Bradford expresó cuantitativamente la relación de proporcionalidad que se daba en la distribución de títulos entre las clases o zonas en que dividió las muestras seleccionadas por él, sobre lubricación y geofísica aplicada, proporcionalidad que formuló matemáticamente con el modelo siguiente: $1: \mathrm{n}: \mathrm{n}^{2}$ (Bradford, 1948 p.116) (véase también Gorbea, 1996, pp. 13 - 31).

Este modelo matemático, aunque hoy representa uno de los modelos clásicos de la bibliometría, puesto que se emplea la mayoría de las veces para identificar la regularidad de la concentración/ dispersión que se observa en los flujos de información documental, tuvo su origen en el interior de una biblioteca y las causas que lo motivaron estaban asociadas a la toma de decisiones en materia de adquisición, pronóstico, planificación de indización y compilación de información bibliográfica.

En este modelo y en otros clásicos de la bibliometría se conjugan los dos comportamientos identificados, anteriormente, en el proceso de la modelación matemática, es decir, el que va dirigido a identificar comportamiento matemático que se traduce en leyes o teorías y el que se orienta a analizar elementos de pronóstico y simulación de determinados procesos y tareas dentro de una biblioteca.

Cabe destacar que la mayoría de las formulaciones matemáticas que se han utilizado en la actividad bibliotecaria han estado orientadas, en sentido general, al pronóstico y planificación de la gestión bibliotecaria y, en particular a la evaluación y pronóstico de la circulación o préstamo de documentos, de las colecciones o fondos de información o al de la eficiencia del sistema bibliotecario, destacándose significativamente los procesos de circulación o préstamo y el de evaluación de colecciones, con el propósito de medir su crecimiento y la intensidad con que éstos se usan.

El creciente interés que ha alcanzado el análisis cuantitativo de las colecciones y la circulación de documentos se debe a que las colecciones, en el sentido más amplio, constituyen la base y el medio fundamental de los servicios bibliotecarios y de información, es el principal recurso que alimenta el resto de los procesos y tareas bibliotecarias y la correspondencia entre la colección y las solicitudes de los usuarios constituye la vía principal para comprobar la efectividad de las colecciones, en función del servicio bibliotecario.

Esta relación o correspondencia que se manifiesta entre la dinámica de las colecciones y el uso que los usuarios hacen de ella ha constituido el centro de preocupación para los que se han adentrado en la modelación matemática de la actividad bibliotecaria. 
Sin embargo, las representaciones matemáticas relacionadas con el estudio de las colecciones y su uso son sólo un tenue ejemplo de todo lo que matemáticos y bibliotecólogos han hecho y pueden seguir haciendo en este ámbito, con el interés de obtener una aproximación cuantitativa a la actividad bibliotecaria, que ayude a la comprensión y pronóstico de todo lo que sucede en el interior de la biblioteca y de su relación con el entorno social al cual pertenece.

Fue precisamente un bibliotecólogo con formación matemática quien al observar el desarrollo que había alcanzado la aplicación de métodos y modelos matemáticos a la actividad bibliotecaria, propuso el nombre de una especialidad que se distinguía dentro del cuerpo de conocimientos de la bibliotecología.

\section{Librametry (Bibliotecometría)}

En 1948 Ranganathan, al comentar una ponencia de Bernal en la Conferencia Anual de la ASLIB en Leamington, Inglaterra, empleó, por primera vez, el término Libramery(Ranganathan, 1969, p. 286).

Este término traducido al español como bibliotecometría ha sido empleado por Morales en 1988 y por Setién y G orbea en 1990, aunque con objetivos diferentes en cada trabajo; en este último se define como:

[...]la aplicación de métodos y modelos matemáticos al estudio de los fenómenos propios de la actividad bibliotecaria con el fin de caracterizar el comportamiento de los componentes que integra la actividad y las tendencias que se presentan en el uso de las bibliotecas y sus fondos.

Este término olvidado en medio de la pirotecnia semántica surgida en los últimos tiempos, en los estudios métricos de la información, es también reivindicado en otro trabajo de Gorbea en 1990, en el que se diferencia de otras especialidades métricas como la Bibliometría, la Informetría, la Cienciometría, pero de forma no hegemónica, al contraponerse al criterio planteado por algunos autores que han pretendido atribuirle a cada uno de estos términos un enfoque absolutista (Gorbea, 1994, p. 24).

En el trabajo antes referido tal diferenciación y definición se sustenta sobre la base de los aspectos señalados por Ranganathan, aunque cabe destacar que él no hizo una definición exacta sobre el término, sin embargo en el análisis e interpretación de sus aplicaciones se puede observar que esta especialidad métrica se refiere a los estudios cuyos resultados contribuyen al desarrollo de la actividad bibliotecaria, en tanto caracterizan el comportamiento de los componentes que integran esta actividad y las tendencias que se presentan en el uso de las bibliotecas y sus colecciones.

Ranganathan identificó que el análisis matemático y estadístico constituía una tecnología clave en el desarrollo y pronóstico de la actividad bibliotecaria, al igual que otras disciplinas científicas que habían desarrollado especialidades tales como la biometría, la econometría, la psicometría, la sociometría, entre otras. 
En 1969, en el Seminario Anual del Centro de D ocumentación para la Investigación y Entrenamiento (DRTC), en Bangalore, India, Ranganathan presentó una discusión sobre cómo había aplicado las técnicas bibliotecométricas en el diseño y organización de diferentes procesos y servicios de la actividad bibliotecaria en la Universidad de Madras.

Su problema inicial consistía en diseñar la construcción de la biblioteca de esta universidad, para lo cual utilizó un enfoque modular, a través del cual cuantificó información referida a los libros, los lectores y el espacio necesario para las funciones de la biblioteca.

En este estudio cuantificó información antropométrica sobre las características de los usuarios, el espacio entre los estantes y el ancho de los mismos, a fin de considerar aspectos tales como la comodidad y postura de los lectores en el diseño de las sillas y las mesas para la sala de lectura, así como la circulación entre los estantes, debido a que consideró el tipo de estantería abierta y los flujos de circulación de documentos y usuarios en los horarios pico. D e esta manera, Ranganathan cuantificó todas y cada una de las variables seleccionadas en su estudio de acuerdo con los requerimientos del sistema de servicio esperado.

No obstante a que Ranganathan acuñó y discutió consistentemente el término Libramłry, como ya se señaló anteriormente, no precisó una definición exacta del mismo. En 1985 Sengupta definió Libramery como "El análisis cuantitativo de los procesos o facetas de la actividad bibliotecaria y de los documentos de la biblioteca, mediante la aplicación del cálculo matemático y estadístico para solucionar los problemas de la biblioteca" (Sengupta, 1992, p.87).

Esta definición apoyada por supuesto en los aspectos que Ranganathan había formulado bajo este término denota que esta especialidad métrica no sólo hace referencia a la actividad bibliotecaria en su conjunto, sino que se ocupa del análisis cuantitativo de todos los procesos y tareas en los que intervienen los documentos en una biblioteca.

Lo anterior se puede constatar a partir del inventario de procesos y tareas, ya que Ranganathan (y relacionadas por el propio Sengupta en el documento de referencia) sostiene que las técnicas bibliotecométricas pueden ser muy provechosas, porque:

1. Determinan la plantilla óptima del personal de la biblioteca y su adecuada distribución en las diferentes secciones.

2. Despliegan al personal de la biblioteca de manera tal que pueda resolver las preguntas de referencia durante los diferentes horarios de la biblioteca.

3. Sistematizan la circulación de los documentos de la biblioteca.

4. D esarrollan y organizan los sistemas de las bibliotecas locales, estatales, regionales y nacionales.

5. D eterminan el tamaño óptimo de un servicio de biblioteca.

6. Diseñan la construcción del edificio de la biblioteca de acuerdo con el tamaño requerido y el mobiliario adecuado. 
7. Distinguen los servicios de las bibliotecas de internados.

8. Analizan las preguntas de los lectores.

9. Modernizan el sistema de adquisición mediante el análisis de los tipos de documentos requeridos en la biblioteca.

10. Determinan la secuencia más satisfactoria para la clasificación de macro y micro documentos.

11. Prueban la exactitud de los registros de los catálogos por las técnicas de muestreo.

12. Aseguran la selección adecuada de los documentos en la biblioteca.

De la observación de estas aplicaciones se puede deducir que los estudios bibliotecométricos ayudan a la generación de nuevos conocimientos sobre la organización y sistematización de los sistemas bibliotecarios, en tanto que constituyen una valiosa herramienta en la medición cuantitativa del costo, la efectividad y el conocido análisis de costo/ beneficio en la inversión que se realiza en la actividad bibliotecaria.

Las aplicaciones métricas de Ranganathan al diseño de los sistemas bibliotecarios demuestran también que la bibliotecometría no sólo se refiere a la evaluación de procesos y tareas de la actividad bibliotecaria, con el interés de identificar el comportamiento económico en el funcionamiento de la misma, sino también que es una importante herramienta en el diseño y arquitectura de edificios y servicios bibliotecarios, en el desarrollo de las colecciones e inclusive del modo en que se organizan los espacios y se distribuyen los muebles para un mejor funcionamiento del sistema.

Pero es bueno reconocer que en la actividad bibliotecaria han quedado un poco olvidadas aquellas aplicaciones métricas que Ranganathan, tan intensivamente, empleó en la biblioteca de la Universidad de Madras, a excepción de un trabajo publicado por Booth en 1969, quien utilizó en un estudio sobre la geometría de las bibliotecas los elementos espaciales propuestos por Ranganathan, pero esta vez considerando la frecuencia de uso de cada libro en la biblioteca (Booth, 1969, p. 28). Para ello, este autor dispuso los libros más prestados a menor distancia del mostrador de préstamo y viceversa, con estos arreglos de distribución espacial y de acuerdo con la frecuencia de uso pudo incrementar la eficiencia en el acceso en diez veces a la existente.

En la actualidad, la tendencia de la modelación matemática a la actividad bibliotecaria se centra en la preocupación por la eficiencia y rentabilidad de los sistemas bibliotecarios, de ahí que la orientación fundamental de este tipo de estudios vaya dirigida a conocer y pronosticar la circulación y uso de los documentos, evaluación y optimación de las colecciones y, por supuesto, el ya mencionado análisis costo/ beneficio entre la inversión de recursos y la satisfacción del usuario en términos de relevancia o pertinencia de la información suministrada, mediante determinado servicio. 
Lo anterior se debe a que en la biblioteca, al igual que en otras esferas de la economía, el almacén, o depósito en este caso, resulta un recurso clave en la gestión de todo servicio, y una de sus principales inversiones. Como ya se señaló anteriormente es la base y el medio para satisfacer las necesidades de los usuarios, por lo que del comportamiento que presente su uso se constituirá la base que pronosticará en cierta medida la eficiencia en la inversión de este recurso, es decir, de la colección y por consiguiente de su correspondencia con las necesidades del lector o usuario.

En este sentido Fussler y Simon en 1969, citados por Burrell y Fenton (1994, p.101) señalan que "la mejor y única forma de pronosticar el uso futuro de los libros es a través del análisis del uso previo que se ha hecho de ellos". Es evidente que para que esto se cumpla, la biblioteca deberá contar con un adecuado y eficiente sistema de información y control sobre el uso de los documentos, así como disponer de estadísticas confiables que garanticen un reflejo objetivo del uso que se hace de los documentos.

La variación en el uso que presentan los libros en una biblioteca, así como los usuarios que los solicitan y usan, hace que los modelos más utilizados en esta evaluación sean los de tipo probabilístico o estocástico, y son aquellos modelos que presentan la peculiaridad de que tanto las variables que forman parte de las ecuaciones, como las de la función objetivo sean variables aleatorias.

Uno de los primeros modelos desarrollados para el estudio del comportamiento del préstamo de documento fue el modelo Markov-Poisson utilizado por Morse en 1968.

En este trabajo, Morse describe con detalle un modelo matemático simple para los procesos de préstamo a partir de supuestos que señalan:

* Elnúmero devecesqueesusado un do cu men to (en unaño) deunadetermi nada clase temática siga una distribución de tipo Poisson.

* El uso que reciben losdo cumen tosaño poraño tienen unaocurren ciaacorde con una cadena de Markov (Burrell, 1986, p.115).

A partir de estos supuestos, Morse formula su modelo conocido como el modelo mezclado de Markov-Piosson, mismo que más tarde es desarrollado por Chen y el propio Morse, en 1976, con el propósito de modificarlo y hacerlo más sencillo, y cuya referencia más cercana, en español, la encontramos en la aplicación que Heshmatallah hizo de este modelo, a la biblioteca del ITAM (Heshmatallah, 1988 p. xiii).

Uno de los autores contemporáneos que más ha estudiado los problemas en la circulación, préstamo y uso de las colecciones es, sin lugar a dudas, Quentin Burrell, quien en uno de sus primeros trabajos propone un modelo estocástico simple para los préstamos de documentos en la biblioteca.

Este modelo lo sugiere el autor a partir de la observación que hace sobre la historia del préstamo externo de un libro en un periodo dado, las variables observadas en su modelo son: a) fecha de su adquisición b) fecha en que es prestado 
por primera vez c) veces que ha sido prestado d) veces que ha sido devuelto e) veces que ha sido solicitado por otros lectores, a los que no se les ha prestado; todas ellas correlacionadas con la variable tiempo son variables en cantidades imprevisibles.

Los resultados de la aplicación de este modelo le ayudan a plantear a Burrell que la distribución de la circulación observada en una biblioteca es de tipo geométrica simple, es decir, según Firthorne, citado por Setién (1991, p. 26) "que la variable independiente crece en forma geométrica y la dependiente en forma aritmética." Ello se debe a que los documentos de una colección tienen diferentes niveles de popularidad y que la distribución de esa popularidad es un exponente negativo. Esta variación negativa de la popularidad conlleva a que la mayoría de los libros lleguen a alcanzar el couso, categoría ésta definida por el propio autor, debido al nivel de obsolescencia que pueden alcanzar los documentos.

La formulación matemática de este modelo puede ser consultada en la versión original del artículo de Burrell (1980, p.119), en el de Hindle and Worthington (1980, p.210), en éste con una propuesta de modelo alternativo al de Burrell, y en el de Setién (1991, p.26), que incluye su aplicación al comportamiento de bibliotecas públicas cubanas y lo presenta como:

D onde:

$$
f r(\mathrm{~T})=f p q^{r} \text { para } r=0,1,2
$$

$f r(\mathrm{~T})=$ Cantidad de volúmenes prestados $r$ veces durante un periodo determinado $\mathrm{T}$;

$f=$ Cantidad total de volúmenes disponibles para el préstamo durante ese periodo;

$p=$ Probabilidad de que los volúmenes utilizados se presten una sola vez cada uno;

$q^{r}=$ Probabilidad de que los volúmenes utilizados se presten rveces cada uno.

Al trabajo de Burrell le sucedieron varios del mismo autor, Burrell (1982), (1985), (1986), (1987), (1988), Brawnsey and Burrell (1986) y Burrell (1990), (1994). Todos dirigidos a conocer el pronóstico sobre la circulación de los documentos en una biblioteca. En cada uno se observan modificaciones al modelo que le antecede, así como la introducción de nuevos conceptos para la medición de este fenómeno, entre los que se pueden mencionar:

* El comportamiento de las distribuciones de frecuencia de la circulación de documentos varía en función del tamaño de éstas y del periodo utilizado (Burrell, 1982).

* Lainfluen ciaqueejerceelenvejecimien to delos do cumen tosen elcompor tamiento de la circulación (Burrell 1985, 1986, 1987). 
Pero no todo lo publicado por Burrell acerca de sus modelos y variaciones ha recibido aceptación entre los especialistas en estos temas; una de las primeras críticas que se hizo sobre su trabajo, la publicaron Hindle y Worthington (1980), en un artículo donde señalan las deficiencias del modelo, mientras que Bagust (1983), en una aplicación que hace de los modelos de Burrell a las bibliotecas públicas de $\mathrm{G}$ ran Bretaña, resume que los modelos de Burrell, sobre la circulación de documentos en una biblioteca, soportan tres proposiciones:

* Quelafrecuen ciadepréstamo deun libro en unaco lec ción esun pro ceso de tipo Poisson.

* Que el comportamiento "deseable" de los libros en una colección está gobernado por determinada distribución probabilística (originalmente exponencial negativa).

* Que cualquier colección puede contener una clase de libros, los cuales pueden ser consideradoscomo mutos en términosdesucirculación(deno mi nada como la dasecro).

Más adelante este autor señala que: "los postulados de Burrell involucran hipótesis que derivaron en conclusiones engañosas, debido a que contrasta información "conveniente" en la construcción de su modelo, lo que le hace caer en su propia trampa" (Bagust, 1983, p.24).

Por su parte, Setién, en el trabajo antes citado (1991, p. 28), demuestra que el modelo matemático de Burrell no se ajusta a los datos de las bibliotecas públicas cubanas, debido a que Burrell considera relativamente estables las tasas de préstamo por volúmenes, similar situación presentan los modelos propuestos por Leimkuhler y Cooper (1971) sobre el crecimiento de los fondos bibliotecarios y las tasas de desuso del fondo por obsolescencia, aspectos éstos que no se presentan en las muestras estudiadas por Setién.

Desde 1971, Leimkuhler y Cooper proponen un conjunto de modelos analíticos para la toma de decisiones en materia de planificación de bibliotecas, mediante el análisis de los aspectos relacionados con el costo, el crecimiento exponencial y la obsolescencia de un fondo bibliotecario y sus efectos sobre su adquisición, almacenamiento y circulación, además de formalizar uno general para el análisis costo/ beneficio de la biblioteca.

En este trabajo, estos autores presentan un modelo (uno de los más citados en materia de modelación matemática de biblioteca) de costo para un sistema de almacenamiento de información, cuya fórmula se representa como:

$$
K(t)=k_{1}+k_{2} t+k_{3} u(t)
$$

Donde:

$K(t)=$ al costo total de almacenamiento de un documento por un periodo $t$ expresado en años. 
$k_{1}=$ al costo inicial de adquisición del documento

$k_{2} t=$ al costo de almacenamiento, el cual se relaciona linealmente con el periodo de retención del documento, sin ser prestado.

$k_{3} u(t)=$ al costo de uso, el cual es proporcional al número de veces que ha sido usado el documento durante el periodo $t$.

Este modelo, al igual que el de Burrell, representa una distribución geométrica simple, en la que la variable independiente, es decir, el costo del almacenamiento, crece geométricamente y la dependiente, el uso, crece aritméticamente. El costo de adquisición y el volumen del fondo almacenado, por lo general, presentan un crecimiento exponencial que encarece el costo de almacenamiento si su uso no se manifiesta en la misma dimensión.

Ese crecimiento exponencial de la colección le sugiere a los autores un modelo simple que puede ser representado como:

$$
N_{t}=N_{0} e^{-\alpha t}
$$

D onde:

$\mathrm{N}_{\mathrm{t}}$ se define como el tamaño del fondo desde un tiempo pasado thasta el tamaño presente $\mathrm{N}_{0}$ y el parámetro $\alpha$ es una constante de crecimiento instantáneo, que se obtiene de la razón existente entre $-N^{\prime} t / N_{t}$

Estos análisis de costo sobre la base del crecimiento y el uso o circulación de los documentos en un fondo bibliotecario los induce a la presentación de un modelo generalizado de análisis costo/ beneficio sobre la operación de toda la biblioteca, en función de la adquisición, el almacenamiento y el uso de los documentos en la misma (véanse más detalles al respecto en Leimkuhler and Cooper, 1971, pp. 390-397).

Un señalamiento general que han recibido estos modelos, además de los ya indicados, sobre el ajuste y generalización de estos modelos, es el relacionado con el nivel de complejidad matemática que presentan, en el sentido de que difícilmente un bibliotecario puede utilizar estas herramientas, si no cuenta con una adecuada preparación en el dominio de las matemáticas.

En ocasiones, la biblioteca y la actividad bibliotecaria en su conjunto han sido objeto de analogías con procesos industriales, empresas de servicios y almacenes inventariados, con una dinámica de entrada y salida similar a la de una colección de biblioteca. Estas observaciones han dado lugar a que especialistas en la modelación matemática, de la economía o la industria, hayan aplicado leyes y modelos estadísticos a la gestión bibliotecaria. 
Un ejemplo de lo anterior es el de Trueswell, quien destacó que la conocida Regla o Ley de distribución estadística 80/ 20, utilizada inicialmente para identificar una regularidad estadística existente en los almacenes de productos industriales, podía ser aplicada también con muy buenos resultados al almacén de biblioteca (todo el fondo de información o una de sus colecciones). En este sentido postuló que:

El 80\% de los movimientos del total de libros que circulan en una biblioteca es satisfecha aproximadamente con el 20\% del total de libros inventariados en esa biblioteca, que aparecen disponibles para su circulación (Trueswell, 1969, p. 458) (véase también Burrell, 1985, p. 24).

Este método que en ocasiones ha sido denominado también como 75/ 25, con la misma interpretación, fue aplicado posteriormente por este autor a los artículos de revistas, para medir aspectos relacionados con la concentración/ dispersión de artículos de revistas.

Asociadas con la analogía entre la biblioteca y la gestión empresarial e industrial, así como al desarrollo alcanzado por los controles económicos y la mercadotecnia, muchas de las interpretaciones y aplicaciones matemáticas a los procesos bibliotecarios, han estado dirigidas hacia la evaluación de la eficiencia, la calidad y el análisis costo/ beneficio que caracterizan el funcionamiento de la actividad bibliotecaria.

El comportamiento de la modelación matemática a la biblioteca ha cubierto una gama muy diversa de métodos y modelos matemáticos y estadísticos, con énfasis en los modelos probabilísticos y estocásticos, así como casi todos los procesos y tareas que se realizan en una biblioteca. Han surgido modelos muy específicos para medir un determinado proceso y otros muy generales para medir la actividad en su conjunto, como el modelo general propuesto por Leimkuhler y Cooper en el trabajo antes citado, sobre el análisis costo/ beneficio de la operación de toda la biblioteca.

Una sustentada crítica, fundamentada en el no ajuste de las muestras utilizadas, sobre el comportamiento de las bibliotecas públicas cubanas, induce a Setién (1995), a proponer un nuevo modelo que se ajuste a esa realidad, muy diferente de las evidencias empíricas observadas, por autores como Burrell y Leimkuhler, en bibliotecas inglesas y que sirvieron para la comprobación de sus modelos.

El modelo propuesto por Setién constituye la referencia más actual en nuestra región sobre la modelación integral de la operación de una biblioteca en desarrollo, en el que se "integran los elementos esenciales de la actividad bibliotecaria, en términos de promedio por bibliotecario" (Setién, 1995, p.14).

Para la argumentación teórica de este modelo de comportamiento de las bibliotecas públicas cubanas y de su índice representativo (IC), Setién se basó en 
propuestas anteriores como las de Rubakin y Medinski, Balika, Stoljarov y la de Rzasa y Baker. Mientras que su justificación matemática la sustenta en lo factible de utilizar los números índices en el análisis del comportamiento de una biblioteca, mediante la comparación de las variables esenciales que describen sus funciones y recursos principales, y de acuerdo con la definición de Spiegel, citado por Setién (1991, p. 29), en tanto que define a los números índices como "una medida estadística que tiene por objeto mostrar los cambios en una variable 0 grupo de variables relacionadas entre sí , con respecto a tiempo, lugares y otras características."

Para más información sobre el origen, desarrollo y formulación matemática de este modelo consultar Setién, 1995 pp. 12-16, véase también el trabajo presentado por el autor en el Seminario-Taller de referencia y publicado en este mismo número.

Entre otras aplicaciones matemáticas, además de la que acabamos de describir, orientadas a evaluar bibliotecas de acuerdo con su tipo, es decir, públicas se puede enmarcar el trabajo ya citado de Bagust (1983) (un modelo para la circulación) y académicas como el de Mitchel $\notin$ al. de 1994, sobre la evaluación de la biblioteca académica.

\section{CONSIDERACIONES FIN ALES}

La aplicación de las matemáticas a las actividades bibliotecaria y de información constituye un nivel de profundización y especialización de los profesionales vinculados a estas actividades, muestra de ello es el surgimiento de especialidades métricas en las ciencias bibliotecológica y de la información como por ejemplo: la bibliometría, la bibliotecometría y la informetría.

La matematización de los fenómenos sociales estudiados por la bibliotecología, como por ejemplo el uso de la información, así como la aplicación de métodos y modelos matemáticos a las actividades bibliotecaria y de información no deberá convertirse en el fin primordial de éstas, al igual que ocurre con la tecnología de cómputo u otra herramienta, sin embargo, el bibliotecólogo o especialista en información que pueda contar con herramientas matemáticas y estadísticas en el estudio o desempeño de sus funciones, ya sea como gestor, docente 0 investigador en esta rama del conocimiento, tendrá ante sí un mundo infinito y casi inexplorado, que de llegar a dominarlo no sólo podrá contar con una información más objetiva para la toma de decisiones, sino que con ello estará contribuyendo también al desarrollo del cuerpo teórico de la disciplina. 


\section{REFERENCIAS BIBLIOGRÁFICAS}

Bagust,A.(1983). "A circulation mo delforbusypubliclibraries". - Jaumal of Doumentation(London)39(1): 24 - 37, March.

Booth, A.D . (1969). "On the geometry of libraries".- Jaumal of Doumentation(London)25(1): 28 - 42, March.

Bradford, S.C. - "The Documentary Chaos", Chapter IX, pp.106-121 In: Documetation - London: Crosby Lockwood and son, Ltd., 1948. - 196 p.

Brownsey, K.W.R. (1986). "Library circulation distributions: some observations on the PLR sample".- Jarmal dDoamenta tion(London)42(1): 22 - 45, March.

Burrell, Quentin (1980). "A simple stochastic model for library loans".- Jaumal of Doumentation (London)36(3): 115 - 132, June.

- - . (1982). "Alternative models for library circulation data." Jaumal of Doumentation(London)38(1): 1 - 13, March.

- -. (1985). "A note on ageing in a library circulation model" .- JaumaldDoamentation(Lon don)41(2): 100 - 115,June.

- -. (1985b). "The 80 / 20 rule: Library lore or statistical law?" - Jaumal ofDoamentation(Lon don)41(1): 24 - 39, March.

- - . (1986). "A se cond note on ageing in ali brary circulation model:Theco rrelation structure" - - JournaldDoamentation(London)42(2): 114-128, June.

- -. (1987). "A third note on ageing in library circulation model: Aplication to futureuseandrelegation".- Jaurnal ofDoamenta tion(London)43(1): 24 - 45, March.

- - and Michael R. Fen ton (1994). "A mo del for li brary book circulation incorporating loan periods".- Jaumal of theAmerian Soiety for Information Saiene (Washington)45(2): 101 - 116, March.

Gó mez,MireyayD ul ceMaPeral ta(1995). "Lasmatemáticasylas ciencias: Su vinculación con los nuevos programas de estudio".- Desded Sur. HumanismoyCienia (México)1(3):37 - 42, diciembre.

G orbeaPortal,Salvador(1994). "Prin cipiosteó ricosymeto do lógicos de los estudios métricos de la información". - Investigt aón Biblictedógia (México)8(17): 23 - 32, julio - diciembre.

G orbeaPortal,Salvador. (1996). ElmodelonatemáticodeBradford Su aplicacónalasrevistaslatimoamericanasdlascienaiasbibliotecolóġayde lainformaaón - México: UNAM, Cen tro Universitario deInvesti gaciones Bibliotecológicas.-152 p. (Monografías 21). 
HerreraRevilla, Ismael (1996). Moddacónnmatemática delossistemas- México: UNAM,Cen tro deInvestigacio nesInterdisciplinariasen Cien ciasyHuma nidades. (Conferencia)

Hindle, A. and D. Worthington (1980). Jaumal of Doametation (London)36(3): 209 - 213, September.

Khorramzadeh, Heshmatallah (1988). Moddos matemáticos Marse Marko. Su aplicaión en biblidecas: Evaluadón de uso de daras monogáficas-México: UNAM, Centro Universitario de Investigaciones Bibliotecológicas. - xvi, 124 p. (Monografías 4).

Leimkuhler, Ferdinand F. and Michael D . Cooper (1971). "Analytical models forlibraryplanning". - JarmaldtheAmericanSodidyforInformationSaience(Wa shington)22(6): 390 - 398, November - D ecember.

Puchet Anyul, Martín(1993). "Aspectosdis cur si vosy di dácticos delas rela cio nesen trematemáticasyCienciasSo ciales" .- ReistaMexicanadeSocole ǵa(México)55(4): 101 - 120, octubre - diciembre.

Ran ganathan, S.R. (1969). "Librametryand its sco pe, pp. 285- 301". 7th Doat motation Reserch Training Centre Amual Seminar 1.-Bangalore: DTRC, 1969.

Redondo Bo tella,Luisa(1990).Lamatematizacoónddconocimientodklatotalidadse dal .- La Habana: Ciencias Sociales.- 210 h. (No impreso).

Sengupta, I.N. (1992). "Bibliometrics, Informetrics, Scientometrics and Librametrics:Anoverview" .- Libri(Co penhagen, D en mark)42(2):75-98.

Setién Quesada,Emilio (1991)."Estado dedesarro llo delasbiblio tecaspúbli cas cubanas: Condiciones para la modelación matemática de su activi dad" . - InvestigacónBiblictedóógica(México)5(11):24-32,julio - diciem bre.

- -.(1995). "Mo delo decom portamien to delasbiblio tecaspúblicascubanas ysuíndicerepresentativo”. - InvestigacónBiblicteedógica(México)9(19):1216, julio - diciembre.

Trueswell, Richard L.(1969). "Somebehavo rial patterns of libraryusers: The 80 / 20 rule". - Wilson Library Bullein(New Y ork) 43(43): 458 - 451, January.

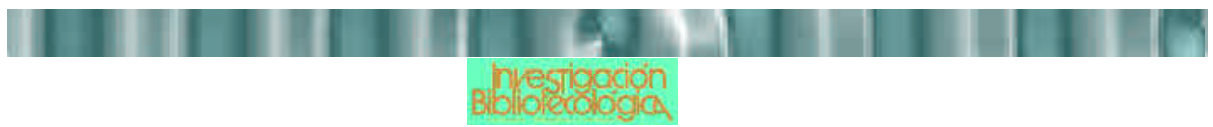

